\title{
THE PHARMACOLOGY OF PRO- AND PREBIOTICS: THE TIME IS NOW
}

\section{F. Visioli}

Department of Molecular Medicine, University of Padua, Padua, Italy

E-mail: francesco.visioli@imdea.org

Doi: $10.36118 /$ pharmadvances.2022.21

We contain more bacteria than cells. Actually, there are far more bacteria than cells. We have bacteria on our skin and inside our body (1). In this respect, human beings can be seen as super-organisms, i.e. a symbiotic co-existence of microbes, fungi, virus, and human cells. There is indeed greater inter-individual variation in the microbiome (or metagenome, that is, the collection of all genomes present in the gut microbiota) than in the human genome. Why did we co-evolve with microbes? Likely, because we guarantee their existence and, in return, they process the food we eat and, for example, they synthesize vitamins and short-chain fatty acids. However, there is much more that shapes our relationship with the bacteria that inhabit our intestine. The most notable example is their participation in the functionality of the immune system. In addition, the gut microbiota is somehow involved in the development (and prevention) of cardiometabolic disorders and cholesterol control (2). For example, although the exact pathways by which intestinal microbiota contribute to obesity remain poorly elucidated, it is becoming quite clear that the lower gut microbiota diversity the higher the risk to develop obesity (3). Furthermore, due to the gut-brain axis, the microbiota participates in the onset of some psychiatric diseases such as depression. In short, there is plenty of room for the development of appropriate and validated probiotics, i.e. bacteria that, via pertinent formulation of pharmaceutical preparations (1).

Taking probiotics is not enough: we need to feed them. Bacteria feed on prebiotics, namely fiber. In short, optimal health can be achieved at least in part through the ingestion of probiotics and prebiotics, to maintain an optimal status of eubiosis.

To summarize, probiotics and prebiotics, as well as nutraceuticals for, e.g., cholesterol control, are today an integral part of pharmacological research and are very often added to pharmacological arsenal (4-6).

This issue of PharmAdvances contains the abstracts and posters presented at the $11^{\text {th }}$ Probiotics, Prebiotics \& New Foods, Nutraceuticals, and 
Botanicals for Nutrition \& Human and Microbiota Health, held in Rome, Italy (12-14/09/2021), and organized by Professors L. Capurso, A. Gasbarrini, A. Guarino, and L. Morelli. The congress was very well-attended, with over 600 participants from all over the world engaging in lively discussions. As you will read in this issue, research in this area is very active and there are plenty of preparations available in the market (6). Some of them have more science behind than other ones, but accumulated and growing evidence indicates an involvement of the intestinal microbiota in cardiovascular risk modulation and in the etiology of other diseases. We still need very large cohort studies that associate particular microbiota profiles with the prevalence of degenerative diseases, notwithstanding residual confounders. Yet, the rapidly evolving field of network medicine, based on big data, machine learning, and artificial intelligence will simplify and speed up data analysis and processing. In the end, next-generation sequencing and metagenomics will be further implemented to provide clinically-useful data (1).

In conclusion, there is plenty of useful information in this issue of PharmAdvances, which links the microbiota with human pathology, opening new avenues for the treatment of degenerative disorders, via proper formulation of validated probiotics, prebiotics, and nutraceuticals derived from the botanical world.

Prof. Francesco Visioli Executive Editor, PharmAdvances

\section{REFERENCES}

1. Scarmozzino F, Poli A, Visioli F. Microbiota and cardiovascular disease risk: A scoping review. Pharmacol Res 2020;159. Doi:104952 10.1016/j.phrs.2020.104952.

2. Illiano P, Brambilla $R$, Parolini $C$. The mutual interplay of gut microbiota, diet and human disease. FEBS J 2020;287(5):833-55. Doi:10.1111/febs.15217.

3. Wolters $M$, Ahrens J, Romani-Perez $M$, et al. Dietary fat, the gut microbiota, and metabolic health - A systematic review conducted within the MyNewGut project. Clin Nutr 2019;38(6):2504-20. Doi:10.1016/j.clnu.2018.12.024.

4. Poli A, Barbagallo CM, Cicero AFG, et al. Nutraceuticals and functional foods for the control of plasma cholesterol levels. An intersociety position paper. Pharmacol Res 2018;134:51-60. Doi: 10.1016/j. phrs.2018.05.015.

5. Poli A, Visioli F. Pharmacology of Nutraceuticals with Lipid Lowering Properties. High Blood Press Cardiovasc Prev 2019;26(2):113-8. Doi:10.1007/s40292-019-00311-x.

6. Delzenne NM, Bindels LB. Food for thought about manipulating gut bacteria. Nature 2020;577(7788):32-4. Doi:10.1038/d41586-019-03704-z. 